\title{
MULTI-PARAMETER ASYMPTOTIC ERROR RESOLUTION OF THE MIXED FINITE ELEMENT METHOD FOR THE STOKES PROBLEM
}

\author{
Ainui Zhou ${ }^{1}$
}

\begin{abstract}
In this paper, a multi-parameter error resolution technique is applied into a mixed finite element method for the Stokes problem. By using this technique and establishing a multi-parameter asymptotic error expansion for the mixed finite element method, an approximation of higher accuracy is obtained by multi-processor computers in parallel.

Résumé. Dans cet article une méthode de résolution d'erreurs multi-paramétrique est appliquée à la méthode des éléments finis mixte pour le problème de Stokes. À l'aide de cette technique, et en utilisant un développement asymptotique multi-paramétrique de l'erreur, on obtient une plus grande précision par calcul parallèle sur des machines multi-processeurs.
\end{abstract}

AMS Subject Classification. 65N15, 65N30.

Received: June 26, 1998. Revised October 21, 1997 and March 3, 1998.

\section{INTRODUCTION}

The Stokes equations have become an important model problem in computational fluid dynamics for designing and analyzing finite element algorithms. In the context of the Galerkin variational formulation, the computational accuracy for the velocity components can be compared to that known for the Poisson equation. For the pressure, however, one frequently observes a drastic reduction of the accuracy along the boundary. Therefore, one has anyway to employ some techniques or arguments to recover and increase the accuracy.

The extrapolation approach established by Richardson in 1926, is an important technique to obtain approximations of higher accuracy. The application of this approach in the finite difference method can be found in [13]. In 1983, Lin, Lü and Shen [10] have applied the technique to the finite element method. Development in this direction can be found in $[3,12,14]$. However, this approach has a limitation since it requires a global refinement and hence wastes computing time and memory. Recently, a so-called multi-parameter error resolution technique has been introduced to obtain approximations of higher accuracy (see [19,20]). This new approach is based on a multi-parameter asymptotic error expansion and only uses partial refinements. It is shown that a combination of discrete solutions related to such partial refinements can produce an approximation of higher accuracy and the procedure can be done by multi-processor computers in parallel. In this paper, we apply this technique to a mixed finite element method for the Stokes problem.

Consider the homogenous Stokes problem

$$
\left\{\begin{array}{rlrl}
-\Delta \mathbf{u}+\nabla p & =\mathbf{f}, & & \text { in } \Omega, \\
\operatorname{div} \mathbf{u}=0, & & \text { in } \Omega, \\
\mathbf{u} & =0, & & \text { on } \partial \Omega,
\end{array}\right.
$$

Key words and phrases. Multi-parameter asymptotic error resolution, multi-parameter extrapolation, mixed finite element, Stoke problem, parallel computing.

${ }^{1}$ Institute of Systems Science, Academia Sinica, Beijing 100080, China. e-mail: azhou@bamboo.iss.ac.cn. 
where $\Omega=[0,1]^{2}, \mathbf{u}=\left(u_{1}, u_{2}\right)$ is the velocity, $p$ is the pressure and $\mathbf{f}$ indicates the external force. We know that (1.1) is equivalent to the variational formulation( [8]): Find $(\mathbf{u}, p) \in\left(H_{0}^{1}(\Omega)\right)^{2} \times L_{0}^{2}(\Omega)$ such that

$$
B(\mathbf{u}, p ; \mathbf{v}, q)=(\mathbf{f}, \mathbf{v}), \quad \forall(\mathbf{v}, q) \in\left(H_{0}^{1}(\Omega)\right)^{2} \times L_{0}^{2}(\Omega),
$$

where

$$
\begin{gathered}
L_{0}^{2}(\Omega)=\left\{q \in L^{2}(\Omega): \int_{\Omega} q=0\right\} \\
B(\mathbf{u}, p ; \mathbf{v}, q)=(\nabla \mathbf{u}, \nabla \mathbf{v})-(p, \operatorname{div} \mathbf{v})+(q, \operatorname{div} \mathbf{u})
\end{gathered}
$$

and other notations are identical to these of references [5, 8].

Divide $\Omega$ into subrectangles $\Omega_{j}(j=1, \cdots, m)$ whose edges are parallel to the $x$-axis and the $y$-axis respectively. On each $\Omega_{j}(j=1, \cdots, m)$ a uniform mesh is imposed, and globally a quasi-uniform partition on $\Omega$ is formed. Denote the mesh sizes on $\Omega_{j}$ by $h_{j, 1}$ in $x$-direction and $h_{j, 2}$ in $y$-direction $(j=1, \cdots, m)$. Among these mesh parameters, some are independent, say $h_{1}, \cdots, h_{s}$. It can be proved that $2 \leq s \leq m+1$ and one can choose $s>2$. Let $h=\max \left\{h_{j}, \cdots, h_{s}\right\}, T_{h}$ be the partition on $\Omega$ and $\left(\mathbf{V}_{h}, L_{h}\right)$ be a pair of finite element spaces on $T_{h}$, e.g. the Bernardi-Raugel finite element space or the filtered bilinear-constant finite element space, satisfying the Babuska-Brezzi conditon (see $[2,8]$ ). The interpolated finite element approximations corresponding to $h_{1}, \cdots, h_{s}$ is denoted by $\left(\mathbf{u}\left(h_{1}, \cdots, h_{s}\right), p\left(h_{1}, \cdots, h_{s}\right)\right)$. Then there exist multi-parameter error resolution expressions on $\Omega$ (see Sect. 4)

$$
\left\{\begin{array}{l}
\mathbf{u}\left(h_{1}, \cdots, h_{s}\right)=\mathbf{u}+\sum_{j=1}^{s} \mathbf{a}_{j} h_{j}^{2}+O\left(h^{3}\right), \text { in } H^{1}-\text { norm } \\
\mathbf{u}\left(h_{1}, \cdots, h_{s}\right)=\mathbf{u}+\sum_{j=1}^{s} \mathbf{a}_{j} h_{j}^{2}+O\left(h^{4}\right), \text { in } L^{2}-\text { norm } \\
p\left(h_{1}, \cdots, h_{s}\right)=p+\sum_{j=1}^{s} b_{j} h_{j}^{2}+O\left(h^{3}\right), \text { in } L^{2}-\text { norm }
\end{array}\right.
$$

where $\mathbf{a}_{j}, b_{j}(j=1, \cdots, s)$ are independent of $\left(h_{1}, \cdots, h_{s}\right)$. Based on (1.3), a multi-parameter extrapolation yields approximations of higher accuracy

$$
\left\{\begin{array}{l}
\left(4 \sum_{j=1}^{s} \mathbf{u}_{j}-(4 s-3) \mathbf{u}_{0}\right) / 3=\mathbf{u}+O\left(h^{3}\right), \text { in } H^{1}-\text { norm } \\
\left(4 \sum_{j=1}^{s} \mathbf{u}_{j}-(4 s-3) \mathbf{u}_{0}\right) / 3=\mathbf{u}+O\left(h^{4}\right), \text { in } L^{2}-\text { norm } \\
\left(4 \sum_{j=1}^{s} p_{j}-(4 s-3) p_{0}\right) / 3=p+O\left(h^{3}\right), \text { in } L^{2}-\text { norm }
\end{array}\right.
$$

where $\left(\mathbf{u}_{0}, p_{0}\right)=\left(\mathbf{u}\left(h_{1}, \cdots, h_{s}\right), p\left(h_{1}, \cdots, h_{s}\right)\right)$ and $\left(\mathbf{u}_{j}, p_{j}\right)=\left(\mathbf{u}\left(h_{1}, \cdots, h_{j-1}, h_{j} / 2, h_{j+1}, \cdots, h_{s}\right)\right.$, $\left.p\left(h_{1}, \cdots, h_{j-1}, h_{j} / 2, h_{j+1}, \cdots, h_{s}\right)\right)$.

Therefore, a parallel algorithm for approximations of higher accuracy can be designed as follows.

\section{Algorithm.}

Step 1. Compute $\left(\mathbf{u}_{j}, p_{j}\right)(0 \leq j \leq s)$ in parallel.

Step 2. Set

$$
\left\{\begin{array}{l}
\mathbf{u}^{c}=\left(4 \sum_{j=1}^{s} \mathbf{u}_{j}-(4 s-3) \mathbf{u}_{0}\right) / 3 \\
p^{c}=\left(4 \sum_{j=1}^{s} p_{j}-(4 s-3) p_{0}\right) / 3
\end{array}\right.
$$




\section{PRELIMINARIES}

For any element $e=\left[x_{e}-h_{e}, y_{e}-k_{e}\right]^{2}$, we define the interpolation operators $\mathbf{i}_{h}=\left(i_{h, 1}, i_{h, 2}\right):(C(e))^{2} \longrightarrow$ $Q_{1,2} \times Q_{2,1}$ and $j_{h}: L^{2}(e) \longrightarrow Q_{0}$ by $(c f .[2,8])$

$$
\left\{\begin{array}{c}
\left(\mathbf{v}-\mathbf{i}_{h} \mathbf{v}\right)\left(x_{e} \pm h_{e}, y_{e} \pm k_{e}\right)=0, \forall \mathbf{v} \in(C(e))^{2}, \\
\int_{\left(x_{e} \pm h_{e}, y_{e}-k_{e}\right)}^{\left(x_{e} \pm h_{e}, y_{e}+k_{e}\right)}\left(\varphi-i_{h, 1} \varphi\right) d y=0, \forall \varphi \in C(e), \\
\int_{\left(x_{e}-h_{e}, y_{e} \pm k_{e}\right)}^{\left(x_{e}+h_{e}, y_{e} \pm k_{e}\right)}\left(\varphi-i_{h, 2} \varphi\right) d x=0, \forall \varphi \in C(e), \\
j_{h} \varphi=\frac{1}{|e|} \int_{e} \varphi, \forall \varphi \in L^{2}(e),
\end{array}\right.
$$

where $Q_{r, n}=\operatorname{span}\left\{x^{i} y^{j}: 0 \leq i \leq r, 0 \leq j \leq n\right\}$ and $Q_{r} \equiv Q_{r, r}$. Introduce two other postprocessing interpolation operators. Let $T_{h}$ be a rectangular partition on $\Omega$ with size $h$. We assume that $T_{h}$ has been obtained from $T_{3 h}$ by dividing each element into nine congruent rectangles. Let $\tau=\cup_{i=1}^{9} e_{i} \in T_{3 h}$ with $e_{i} \in T_{h}$, and $\mathbf{I}_{3 h}=\left(I_{3 h, 1}, I_{3 h, 2}\right)$ and $J_{3 h}$ are defined as

$$
\begin{gathered}
\left\{\begin{array}{l}
\left.I_{3 h, j} w\right|_{\tau} \in Q_{3}(\tau), j=1,2, \\
I_{3 h, j} w\left(p_{i}\right)=w\left(p_{i}\right), \quad i=1, \cdots, 16, j=1,2,
\end{array}\right. \\
\left\{\begin{array}{l}
\left.J_{3 h} p\right|_{\tau} \in Q_{2}(\tau), \\
\int_{e_{i}}\left(J_{3 h} p-p\right)=0, \quad i=1, \cdots, 9,
\end{array}\right.
\end{gathered}
$$

where $p_{i}(i=1, \cdots, 16)$ are all vertices of $e_{1}, \cdots, e_{9}$. It can be proved that $(c f .[11,17,18])$

$$
\begin{aligned}
& \left\{\begin{array}{l}
\mathbf{I}_{3 h} \mathbf{i}_{h}=\mathbf{I}_{3 h}, \\
\left\|\mathbf{I}_{3 h} \mathbf{v}\right\|_{1} \leq c\|\mathbf{v}\|_{1}, \quad \forall \mathbf{v} \in \mathbf{V}_{h}, \\
h^{-1}\left\|\mathbf{I}_{3 h} \mathbf{w}-\mathbf{w}\right\|_{0}+\left\|\mathbf{I}_{3 h} \mathbf{w}-\mathbf{w}\right\|_{1} \leq c h^{r}\|\mathbf{w}\|_{1+r}, \quad \forall \mathbf{w} \in H^{1+r}(\Omega), 1 \leq r \leq 3
\end{array}\right. \\
& \qquad\left\{\begin{array}{l}
J_{3 h} j_{h}=J_{3 h}, \\
\left\|J_{3 h} q\right\|_{0} \leq c\|q\|_{0}, \quad \forall q \in L_{h}, \\
\left\|J_{3 h} w-w\right\|_{0} \leq c h^{r}\|w\|_{r}, \quad \forall w \in H^{r}(\Omega), 1 \leq r \leq 3 .
\end{array}\right.
\end{aligned}
$$

In order to solve the problem (1.1), many pairs of finite element spaces were introduced $($ see $[2,8])$. One of them is the so-called Bernardi-Raugel element, which is defined by

$$
\left\{\begin{array}{l}
\mathbf{V}_{h}=\left\{\mathbf{v} \in(C(\bar{\Omega}))^{2},\left.\quad \mathbf{v}\right|_{e} \in Q_{1,2}(e) \times Q_{2,1}(e),\left.\mathbf{v}\right|_{\partial \Omega}=0, e \in T_{h}\right\} \\
L_{h}=\left\{q \in L_{0}^{2}(\Omega),\left.\quad q\right|_{e} \in Q_{0}(e), \quad \int_{\Omega} q=0, e \in T_{h}\right\}
\end{array}\right.
$$

We mention here that the Bernardi-Raugel element was presented but not enalysed in [7]. 


\section{ERror RESOLUTION FOR INTERPOLANTS}

We have the following multi-parameter error resolution for interpolants.

Theorem 3.1. For $(\mathbf{u}, p) \in\left(H^{4}(\Omega)\right)^{2} \times H^{3}(\Omega)$, there exist $\mathbf{f}_{j} \in\left(L^{2}(\Omega)\right)^{2}, \mathbf{g}_{j} \in\left(H^{1 / 2}(\Gamma)\right)^{2}(j=1, \cdots, s)$ such that

$$
\begin{aligned}
& B\left(\mathbf{u}-i_{h} \mathbf{u}, p-j_{h} p ; \mathbf{v}, q\right) \\
= & \sum_{j=1}^{s} h_{j}^{2}\left(\int_{\Omega} \mathbf{f}_{j} \mathbf{v}+\int_{\Gamma} \mathbf{g}_{j} \mathbf{v}\right)+O\left(h^{3}\right)\left(\|\mathbf{u}\|_{4}+\|p\|_{3}\right)\|\mathbf{v}\|_{1}, \forall(\mathbf{v}, q) \in \mathbf{V}_{h} \times L_{h},
\end{aligned}
$$

where $\Gamma=\cup_{1 \leq i, j \leq s} \partial \Omega_{i} \cap \partial \Omega_{j}$.

Proof. Since

$$
\left(q, \operatorname{div}\left(\mathbf{u}-\mathbf{i}_{h} \mathbf{u}\right)\right)=0, \quad \forall q \in L_{h},
$$

it is only necessary to prove two expansions

$$
\left(\nabla\left(\mathbf{u}-i_{h} \mathbf{u}\right), \nabla \mathbf{v}\right)=\frac{1}{3} \sum_{e \in T_{h}} \int_{e}\left(h_{e}^{2} \partial_{x x y y} u_{1}, k_{e}^{2} \partial_{x x y y} u_{2}\right) \mathbf{v}+O\left(h^{3}\right)\|\mathbf{u}\|_{4}\|\mathbf{v}\|_{1}
$$

for $\mathbf{v}=\left(v_{1}, v_{2}\right) \in \mathbf{V}_{h}$, and

$$
\begin{aligned}
\left(p-j_{h} p, \operatorname{div} \mathbf{v}\right)= & \frac{1}{3} \sum_{e \in T_{h}} \int_{e}\left(k_{e}^{2} \partial_{x y y} p, h_{e}^{2} \partial_{x x y} p\right) \mathbf{v}-\frac{1}{3} \sum_{s_{e, 4} \cup s_{e, 2} \subset \Gamma_{x}} k_{e}^{2}\left(\int_{s_{e, 4}}-\int_{s_{e, 2}}\right) \partial_{x y} p v_{1} \\
& -\frac{1}{3} \sum_{s_{e, 3} \cup s_{e, 1} \subset \Gamma_{y}} h_{e}^{2}\left(\int_{s_{e, 3}}-\int_{s_{e, 1}}\right) \partial_{x y} p v_{2}+O\left(h^{3}\right)\|p\|_{3}\|\mathbf{v}\|_{1},
\end{aligned}
$$

where $\Gamma_{x}, \Gamma_{y}$ is the part of $\Gamma$ parallel to the $x$-direction and the $y$-direction respectively, and

$$
\begin{aligned}
& s_{e, 1}=\left\{(x, y): x=x_{e}-h_{e}, y_{e}-k_{e} \leq y \leq y_{e}+k_{e}\right\} \\
& s_{e, 2}=\left\{(x, y): x_{e}-h_{e} \leq x \leq x_{e}+h_{e}, y=y_{e}-k_{e}\right\} \\
& s_{e, 3}=\left\{(x, y): x=x_{e}+h_{e}, y_{e}-k_{e} \leq y \leq y_{e}+k_{e}\right\} \\
& s_{e, 4}=\left\{(x, y): x_{e}-h_{e} \leq x \leq x_{e}+h_{e}, y=y_{e}+k_{e}\right\} .
\end{aligned}
$$

We employ the technique in $[11,17,18]$ and introduce the two error functions

$$
F \equiv F_{e}=\frac{1}{2}\left(\left(y-y_{e}\right)^{2}-k_{e}^{2}\right), \quad E \equiv E_{e}=\frac{1}{2}\left(\left(x-x_{e}\right)^{2}-h_{e}^{2}\right),
$$

then, it is obvious that $F(y)=0$ on $s_{e, 2}$ and $s_{e, 4}, E(x)=0$ on $s_{e, 1}$ and $s_{e, 3}$.

$$
\left\{\begin{aligned}
E & =-\frac{1}{3} h_{e}^{2}+\frac{1}{6}\left(E^{2}\right)_{x x}, \\
x-x_{e} & =\frac{1}{6}\left(E^{2}\right)_{x x x} \\
\left(x-x_{e}\right)^{2} & =\frac{1}{45}\left(E^{3}\right)_{x x x x}+\frac{1}{5} h_{e}^{2}
\end{aligned}\right.
$$


Let $\mathbf{u}=\left(u_{1}, u_{2}\right)$, we only need to go to prove

$$
\left\{\begin{aligned}
F & =-\frac{1}{3} k_{e}^{2}+\frac{1}{6}\left(F^{2}\right)_{y y}, \\
y-y_{e} & =\frac{1}{6}\left(F^{2}\right)_{y y y}, \\
\left(y-y_{e}\right)^{2} & =\frac{1}{45}\left(F^{3}\right)_{y y y y}+\frac{1}{5} k_{e}^{2} .
\end{aligned}\right.
$$

$$
\left(\nabla\left(u_{1}-i_{h, 1} u_{1}\right), \nabla v_{1}\right)=\frac{1}{3} \sum_{e \in T_{h}} h_{e}^{2} \int_{e} \partial_{x x y y} u_{1} v_{1}+O\left(h^{3}\right)\|\mathbf{u}\|_{4}\|\mathbf{v}\|_{1} .
$$

A Taylor expansion yields

$$
\partial_{x} v_{1}=\partial_{x} v_{1}\left(x, y_{e}\right)+\frac{1}{6}\left(F^{2}\right)_{y y y} \partial_{x y} v_{1}\left(x, y_{e}\right)+\left(\frac{1}{90}\left(F^{3}\right)_{y y y y}+\frac{1}{10} k_{e}^{2}\right) \partial_{x y y} v_{1} .
$$

The definition (2.1), integration by parts and inverse estimates lead to

$$
\begin{aligned}
\int_{e} \partial_{x}\left(u_{1}-i_{h, 1} u_{1}\right) \partial_{x} v_{1}\left(x, y_{e}\right) & =0 \\
& = \\
\frac{1}{6} \int_{e}\left(F^{2}\right)_{y y y} \partial_{x}\left(u_{1}-i_{h, 1} u_{1}\right) \partial_{x y} v_{1}\left(x, y_{e}\right) & -\frac{1}{6} \int_{e} F^{2} \partial_{x y y y} u_{1} \partial_{x y} v_{1}\left(x, y_{e}\right) \\
= & O\left(h^{3}\right)\|\mathbf{u}\|_{4}\left\|_{\mathbf{v}}\right\|_{1}, \\
\int_{e} \partial_{x}\left(u_{1}-i_{h, 1} u_{1}\right)\left(\frac{1}{90}\left(F^{3}\right)_{y y y y}+\frac{1}{10} k_{e}^{2}\right) \partial_{x y y} v_{1} & =-\frac{1}{90} \int_{e}\left(F^{3}\right)_{y} \partial_{x y y y} u_{1} \partial_{x y y} v_{1} \\
& =O\left(h^{3}\right)\|\mathbf{u}\|_{4}\|\mathbf{v}\|_{1} .
\end{aligned}
$$

Thus, we obtain

$$
\left(\partial_{x}\left(u_{1}-i_{h, 1} u_{1}\right), \partial_{x} v_{1}\right)=O\left(h^{3}\right)\|\mathbf{u}\|_{4}\|\mathbf{v}\|_{1}
$$

For $\left(\partial_{y}\left(u_{1}-i_{h, 1} u_{1}\right), \partial_{y} v_{1}\right)$, using the definition (2.1),

$$
\partial_{y} v_{1}=E_{x x} \partial_{y} v_{1}\left(x_{e}, y\right)+\frac{1}{6}\left(E^{2}\right)_{x x x} \partial_{x y} v_{1}
$$

and integration by parts,

$$
\begin{aligned}
& \int_{e} \partial_{y}\left(u_{1}-i_{h, 1} u_{1}\right)(E)_{x x} \partial_{y} v_{1}\left(x_{e}, y\right) \\
= & \left(\int_{s_{e, 3}}-\int_{s_{e, 1}}\right) E_{x} \partial_{y}\left(u_{1}-i_{h, 1} u_{1}\right) \partial_{y} v_{1}\left(x_{e}, y\right) d y-\int_{e} E_{x} \partial_{x y}\left(u_{1}-i_{h, 1} u_{1}\right) \partial_{y} v_{1}\left(x_{e}, y\right) \\
= & -\left(\int_{s_{e, 3}}-\int_{s_{e, 1}}\right) E_{x}\left(u_{1}-i_{h, 1} u_{1}\right) \partial_{y y} v_{1}\left(x_{e}, y\right) d y+\int_{e} E \partial_{x x y} u_{1} \partial_{y} v_{1}\left(x_{e}, y\right) \\
= & -\frac{1}{3} h_{e}^{2} \int_{e} \partial_{x x y} u_{1}\left(\partial_{y} v_{1}-E_{x} \partial_{x y} v_{1}\right)+\frac{1}{6} \int_{e}\left(E^{2}\right)_{x x} \partial_{x x y} u_{1} \partial_{y} v\left(x_{e}, y\right) \\
= & -\frac{1}{3} h_{e}^{2} \int_{e} \partial_{x x y} u_{1} \partial_{y} v_{1}+\int_{e} \partial_{x x x y} u_{1}\left(-\frac{1}{3} h_{e}^{2} E \partial_{x y} v_{1}-\frac{1}{6}\left(E^{2}\right)_{x} \partial_{y} v_{1}+\frac{1}{6}\left(E^{2}\right)_{x} E_{x} \partial_{x y} v_{1}\right) \\
= & -\frac{1}{3} h_{e}^{2}\left(\int_{s_{e, 4}}-\int_{s_{e, 2}}\right) \partial_{x x y} u_{1} v_{1} d x+\frac{1}{3} h_{e}^{2} \int_{e} \partial_{x x y y} u_{1} v_{1}+O\left(h^{3}\right)\|\mathbf{u}\|_{4}\|\mathbf{v}\|_{1},
\end{aligned}
$$




$$
\begin{aligned}
& \frac{1}{6} \int_{e}\left(E^{2}\right)_{x x x} \partial_{y}\left(u_{1}-i_{h} u_{1}\right) \partial_{x y} v_{1} \\
& =\frac{1}{6}\left(\int_{s_{e, 3}}-\int_{s_{e, 1}}\right)\left(E^{2}\right)_{x x} \partial_{y}\left(u_{1}-i_{h} u_{1}\right) \partial_{x y} v_{1}-\frac{1}{6} \int_{e}\left(E^{2}\right)_{x x} \partial_{x y}\left(u_{1}-i_{h, 1} u_{1}\right) \partial_{x y} v_{1} \\
& =-\frac{1}{6}\left(\int_{s_{e, 3}}-\int_{s_{e, 1}}\right)\left(E^{2}\right)_{x x}\left(u_{1}-i_{h} u_{1}\right) \partial_{x y y} v_{1}+\frac{1}{6} \int_{e}\left(E^{2}\right)_{x} \partial_{x x y} u_{1} \partial_{x y} v_{1} \\
& =\frac{1}{6} \int_{e}\left(E^{2}\right)_{x} \partial_{x x y} u_{1} \partial_{x y} v_{1} \\
& =O\left(h^{3}\right)\|\mathbf{u}\|_{4}\|\mathbf{v}\|_{1} .
\end{aligned}
$$

Therefore, we have

$$
\begin{aligned}
\left(\partial_{y}\left(u_{1}-i_{h} u_{1}\right), \partial_{y} v_{1}\right)= & \frac{1}{3} \sum_{e \in T_{h}} h_{e}^{2}\left(\left(\int_{s_{e, 4}}-\int_{s_{e, 2}}\right) \partial_{x x y} u_{1} v_{1} d x+\int_{e} \partial_{x x y y} u_{1} v_{1}\right) \\
& +O\left(h^{3}\right)\|\mathbf{u}\|_{4}\|\mathbf{v}\|_{1}
\end{aligned}
$$

By the quasi-uniformity of $T_{h}$, one sees that line integrals in (3.5) disappear during the summation, thus (3.3) follows from $(3.4,3.5)$.

We turn to prove (3.2). Similar to the proof of (3.1), we only need to prove

$$
\begin{aligned}
\left(p-j_{h} p, \partial_{x} v_{1}\right)= & \frac{1}{3} \sum_{e \in T_{h}} k_{e}^{2} \int_{e} \partial_{x y y} p v_{1}-\frac{1}{3} \sum_{s_{e, 4} \cup s_{e, 2} \subset \Gamma_{x}} k_{e}^{2}\left(\int_{s_{e, 4}}-\int_{s_{e, 2}}\right) \partial_{x y} p v_{1} \\
& +O\left(h^{3}\right)\|p \mid\|\left\|_{3}\right\| \mathbf{v} \|_{1} .
\end{aligned}
$$

By the Taylor expansion, we have

$$
\begin{aligned}
\int_{e}\left(p-j_{h} p\right) \partial_{x} v_{1} & =\int_{e}\left(p-j_{h} p\right)\left(\partial_{x} v_{1}\left(x, y_{e}\right)+F_{y} \partial_{x y} v_{1}\left(x, y_{e}\right)+\frac{1}{6}\left(\left(F^{2}\right)_{y y}+k_{e}^{2}\right) \partial_{x y y} v_{1}\right) \\
& \equiv I+I I+I I I .
\end{aligned}
$$

The definition (2.2) shows that $I=0$ and

$$
\begin{aligned}
I I= & -\int_{e} F \partial_{y} p \partial_{x y} v_{1}\left(x, y_{e}\right) \\
= & \frac{1}{3} k_{e}^{2} \int_{e} \partial_{y} p \partial_{x y} v_{1}\left(x, y_{e}\right)-\frac{1}{6} \int_{e}\left(F^{2}\right)_{y y} \partial_{y} p \partial_{x y} v_{1}\left(x, y_{e}\right) \\
= & \frac{1}{3} k_{e}^{2}\left(\int_{s_{e, 3}}-\int_{s_{e, 1}}\right) \partial_{y} p \partial_{y} v_{1}\left(x, y_{e}\right) d y-\frac{1}{3} k_{e}^{2} \int_{e} \partial_{x y} p\left(\partial_{y} v_{1}-F_{y} \partial_{y y} v_{1}\right)+O\left(h^{3}\right)\|p\|_{3}\|\mathbf{v}\|_{1} \\
= & \frac{1}{3} k_{e}^{2}\left(\int_{s_{e, 3}}-\int_{s_{e, 1}}\right) \partial_{y} p \partial_{y} v_{1}\left(x, y_{e}\right) d y-\frac{1}{3} k_{e}^{2} \int_{e} \partial_{x y} p \partial_{y} v_{1}+O\left(h^{3}\right)\|p\|_{3}\left\|_{\mathbf{v}}\right\|_{1} \\
= & \frac{1}{3} k_{e}^{2}\left(\int_{s_{e, 3}}-\int_{s_{e, 1}}\right) \partial_{y} p \partial_{y} v_{1}\left(x, y_{e}\right) d y-\frac{1}{3} k_{e}^{2}\left(\int_{s_{e, 4}}-\int_{s_{e, 2}}\right) \partial_{x y} p v_{1} d x \\
& +\frac{1}{3} k_{e}^{2} \int_{e} \partial_{x y y} p v_{1}+O\left(h^{3}\right)\|p\|_{3}\|\mathbf{v}\|_{1},
\end{aligned}
$$




$$
\begin{aligned}
I I I & =\frac{1}{6} \int_{e} F^{2} \partial_{y y} p \partial_{x y y} v_{1} \\
& =\frac{1}{6}\left(\int_{s_{e, 3}}-\int_{s_{e, 1}}\right) F^{2} \partial_{y y} p \partial_{y y} v_{1} d y-\frac{1}{6} \int_{e} F^{2} \partial_{x y y} p \partial_{y y} v_{1} \\
& =\frac{1}{6}\left(\int_{s_{e, 3}}-\int_{s_{e, 1}}\right) F^{2} \partial_{y y} p \partial_{y y} v_{1} d y+O\left(h^{3}\right)\|p\|_{3}\|\mathbf{v}\|_{1} .
\end{aligned}
$$

Similarly, line integrals along $y$-direction in the above terms will disappear during the summation of $e \in T_{h}$. Thus we complete the proof.

From the proof of Theorem 3.1, we can also obtain the following

Theorem 3.2. For $(\mathbf{u}, p) \in\left(H^{4}(\Omega)\right)^{2} \times H^{3}(\Omega)$, there exist $\mathbf{f}_{j} \in\left(L^{2}(\Omega)\right)^{2}, \mathbf{g}_{j} \in\left(H^{1 / 2}(\Gamma)\right)^{2}(j=1, \cdots, s)$ such that

$$
\begin{aligned}
B\left(\mathbf{u}-i_{h} \mathbf{u}, p-j_{h} p ; \mathbf{v}, q\right)= & \sum_{j=1}^{s} h_{j}^{2}\left(\int_{\Omega} \mathbf{f}_{j} \mathbf{v}+\int_{\Gamma} \mathbf{g}_{j} \mathbf{v}\right) \\
& +O\left(h^{4}\right)\left(\|\mathbf{u}\|_{4}+\|p\|_{3}\right)\left(h^{-1}\|\mathbf{v}-\mathbf{w}\|_{1}+\|\mathbf{w}\|_{2}\right), \forall(\mathbf{v}, q) \in \mathbf{V}_{h} \times L_{h}
\end{aligned}
$$

where $\mathbf{w} \in\left(H_{0}^{1}(\Omega) \cap H^{4}(\Omega)\right)^{2}$.

\section{ERror RESOLUTION FOR FINITE ELEMENT SOLUTIONS}

A mixed finite element method based on (1.1) reads as follows: Find $\left(\mathbf{u}_{h}, p_{h}\right) \in \mathbf{V}_{h} \times L_{h}$ such that

$$
B\left(\mathbf{u}_{h}, p_{h} ; \mathbf{v}, q\right)=(\mathbf{f}, \mathbf{v}), \quad \forall(\mathbf{v}, q) \in \mathbf{V}_{h} \times L_{h} .
$$

or

$$
B\left(\mathbf{u}_{h}-u, p_{h}-p ; \mathbf{v}, q\right)=0, \quad \forall(\mathbf{v}, q) \in \mathbf{V}_{h} \times L_{h}
$$

One has the following error estimate (see $[2,8])$

$$
h^{-1}\left\|\mathbf{u}-\mathbf{u}_{h}\right\|_{0}+\left\|\mathbf{u}-\mathbf{u}_{h}\right\|_{1}+\left\|p-p_{h}\right\|_{0} \leq \operatorname{ch}\left(\|\mathbf{u}\|_{2}+\|p\|_{1}\right)
$$

Now, we shall present the multi-parameter error resolution for the numerical solution.

Theorem 4.1. If $(\mathbf{u}, p) \in\left(H_{0}^{1}(\Omega) \cap H^{4}(\Omega)\right)^{2} \times\left(L_{0}^{2}(\Omega) \cap H^{3}(\Omega)\right)$ is the exact solution of (1.1), then there exist $\left(\mathbf{a}_{j}, b_{j}\right) \in\left(H_{0}^{1}(\Omega) \cap H^{2}(\Omega \backslash \Gamma)\right)^{2} \times\left(L_{0}^{2}(\Omega) \cap H^{1}(\Omega \backslash \Gamma)\right)(j=1, \cdots, s)$ such that

$$
\left\{\begin{array}{l}
\mathbf{I}_{3 h} \mathbf{u}_{h}-\mathbf{u}=\sum_{j=1}^{s} \mathbf{a}_{j} h_{j}^{2}+O\left(h^{3}\right), \text { in } H^{1}(\Omega \backslash \Gamma) \\
J_{3 h} p_{h}-p=\sum_{j=1}^{s} b_{j} h_{j}^{2}+O\left(h^{3}\right), \text { in } L^{2}(\Omega \backslash \Gamma)
\end{array}\right.
$$

Proof. Let $\left(\mathbf{a}_{j}, b_{j}\right) \in\left(H_{0}^{1}(\Omega)\right)^{2} \times L_{0}(\Omega)(j=1, \cdots, s)$ satisfy

$$
B\left(\mathbf{a}_{j}, b_{j} ; \mathbf{v}, q\right)=\left(f_{j}, \mathbf{v}\right)+\int_{\Gamma} \mathbf{g}_{j} \mathbf{v}, \forall(\mathbf{v}, q) \in\left(H_{0}^{1}(\Omega)\right)^{2} \times L_{0}^{2}(\Omega)
$$

and $\left(\mathbf{a}_{j, h}, b_{j, h}\right) \in \mathbf{V}_{h} \times L_{h}(j=1, \cdots, s)$

$$
B\left(\mathbf{a}_{j, h}, b_{j, h} ; \mathbf{v}, q\right)=\left(f_{j}, \mathbf{v}\right)+\int_{\Gamma} \mathbf{g}_{j} \mathbf{v}, \forall(\mathbf{v}, q) \in \mathbf{V}_{h} \times L_{h},
$$


then we have

$$
B\left(\mathbf{u}-i_{h} \mathbf{u}-\sum_{j=1}^{s} \mathbf{a}_{j, h} h_{j}^{2}, p-j_{h} p-\sum_{j=1}^{s} b_{j, h} h_{j}^{2} ; \mathbf{v}, q\right)=O\left(h^{3}\right)\|\mathbf{v}\|_{1}
$$

Taking $\mathbf{v}=\mathbf{u}_{h}-\mathbf{i}_{h} \mathbf{u}-\sum_{j=1}^{s} \mathbf{a}_{j, h} h_{j}^{2}$ and $q=p_{h}-j_{h} p-\sum_{j=1}^{s} b_{j, h} h_{j}^{2}$ in the above equation, we obtain by (4.2) and the Babuska-Brezzi condition (see e.g. $[1,2,4]$ )

$$
\left\{\begin{array}{l}
\mathbf{u}_{h}-\mathbf{i}_{h} \mathbf{u}=\sum_{j=1}^{s} \mathbf{a}_{j, h} h_{j}^{2}+O\left(h^{3}\right), \text { in } H^{1}(\Omega), \\
p_{h}-j_{h} p=\sum_{j=1}^{s} b_{j, h} h_{j}^{2}+O\left(h^{3}\right), \text { in } L^{2}(\Omega) .
\end{array}\right.
$$

Regularity results imply that $\left(\mathbf{a}_{j}, b_{j}\right) \in\left(H_{0}^{1}(\Omega) \cap H^{2}(\Omega \backslash \Gamma)\right)^{2} \times\left(L_{0}^{2}(\Omega) \times H^{1}(\Omega \backslash \Gamma)\right)(j=1, \cdots, s)$ (see [9]) and a similar estimate to (4.3) yields

$$
\left\|\mathbf{a}_{j}-\mathbf{a}_{j, h}\right\|_{1}+\left\|b_{j}-b_{j, h}\right\|_{0} \leq \operatorname{ch}\left(\left\|\mathbf{a}_{j}\right\|_{2}^{\prime}+\left\|b_{j}\right\|_{1}^{\prime}\right), j=1, \cdots, s,
$$

where $\|\cdot\|_{i}^{\prime}$ means piecewise $H^{i}-\operatorname{norm}(\mathrm{i}=1,2)$. Thus, (4.7) together with $(2.5,2.6$, and 4.6) complete the proof.

Theorem 4.2. If $(\mathbf{u}, p) \in\left(H_{0}^{1}(\Omega) \cap H^{4}(\Omega)\right)^{2} \times\left(L_{0}^{2}(\Omega) \cap H^{3}(\Omega)\right)$ is the exact solution of (1.1), then there exist $\mathbf{a}_{j} \in\left(H_{0}^{1}(\Omega) \cap H^{2}(\Omega \backslash \Gamma)\right)^{2}(j=1, \cdots, s)$ such that

$$
\mathbf{I}_{3 h} \mathbf{u}_{h}-\mathbf{u}=\sum_{j=1}^{s} \mathbf{a}_{j} h_{j}^{2}+O\left(h^{4}\right), \text { in } L^{2}(\Omega \backslash \Gamma) .
$$

Proof. Let $\mathbf{a}_{j}, b_{j}, \mathbf{a}_{j, h}$ and $b_{j, h}$ satisfy $(4.4,4.5)$ and set $\eta=\mathbf{u}_{h}-\mathbf{i}_{h} \mathbf{u}-\sum_{j=1}^{s} \mathbf{a}_{j, h} h_{j}^{2}$ as well as $\xi=p_{h}-j_{h} p-$ $\sum_{j=1}^{s} b_{j, h} h_{j}^{2}$, then there exists $(\mathbf{w}, \varphi) \in\left(H_{0}^{1}(\Omega) \cap H^{2}(\Omega)\right)^{2} \times\left(L_{0}^{2}(\Omega) \cap H^{1}(\Omega)\right)$ satisfying

$$
\left\{\begin{array}{l}
B(\mathbf{w}, \varphi ; \mathbf{v}, q)=(\eta, \mathbf{v}), \quad \forall(\mathbf{v}, q) \in\left(H_{0}^{1}(\Omega)\right)^{2} \times L_{0}^{2}(\Omega), \\
\|\mathbf{w}\|_{2}+\|\varphi\|_{1} \leq c\|\eta\|_{0} .
\end{array}\right.
$$

Thus

$$
\begin{aligned}
\|\eta\|_{0}^{2} & =B(\mathbf{w}, \varphi ; \eta, \xi) \\
& =B\left(\mathbf{w}-\mathbf{i}_{h} \mathbf{w}, \varphi-j_{h} \varphi ; \eta, \xi\right)+B\left(\eta, \xi ; \mathbf{i}_{h} \mathbf{w},-j_{h} \varphi\right) .
\end{aligned}
$$

From Theorem 3.1, Theorem 3.2, $(4.6,4.9)$, we have

$$
\begin{aligned}
\left|B\left(\mathbf{w}-\mathbf{i}_{h} \mathbf{w}, \varphi-j_{h} \varphi ; \eta, \xi\right)\right| & \leq \operatorname{ch}\left(\|\mathbf{w}\|_{2}+\|\varphi\|_{1}\right)\left(\|\eta\|_{1}+\|\xi\|_{0}\right) \\
& \leq h^{4}\|\eta\|_{0}
\end{aligned}
$$

and

$$
\begin{aligned}
\left|B\left(\eta, \xi ; \mathbf{i}_{h} \mathbf{w},-j_{h} \varphi\right)\right| & \leq c h^{4}\left(h^{-1}\left\|\mathbf{w}-\mathbf{i}_{h} \mathbf{w}\right\|_{1}+\|\mathbf{w}\|_{2}\right) \\
& \leq c h^{4}\|\mathbf{w}\|_{2} \\
& \leq c h^{4}\|\eta\|_{0} .
\end{aligned}
$$

Combining $(2.5,4.10,4.11)$ and $(4.12)$, we achieve the proof. 


\section{REMARKS}

Only the multi-paramemter error resolution for the Bernardi-Raugel finite element spaces has been discussed above. In fact, the error resolution arguments presented in this paper can be applied to other pairs of finite element spaces with similar results, for instance, the filtered bilinear-constant finite element spaces. Moreover, if the Green-function technique as in [6] is adapted, then maximum norm estimates can also be obtained.

The author would like to express his appreciation to the referees for careful corrections and suggesting several improvements. This work was supported in part by the Chinese National Science Foundation and the Educational Center for Mathematical Research sponsored by the Ministry of Education of China.

\section{REFERENCES}

[1] I. Babuska, The finite element methods with Lagrangian multipliers. Numer. Math. 20 (1973) 179-192.

[2] C. Bernardi and G. Raugel, Analysis of some finite elements of the Stokes problem. Math. Comp. 44 (1985) 71-79.

[3] H. Blum, Asymptotic error expansion and defect correction in the finite element method. Heidelberg (1990).

[4] F. Brezzi, On the existence, uniqueness and approximation of saddle-point problems arising from Lagrangian multipliers. RAIRO Modèl. Math. Anal. Numer. 2 (1974) 129-151.

[5] P.G. Ciarlet, The Finite Element Method for Elliptic Problems. North-Holland (1978).

[6] R. Duran, R.H. Nochetto and J. Wang, Sharp maximum norm error estimates for finite element approximation for the Stokes problem in 2-d. Math. Comp. 51 (1988) 491-506.

[7] M. Fortin, Old and new elements for incompressible flows. Int. J. Numer. Meth. Fluids 1 (1981) 347-367.

[8] V. Girault and P.A. Raviart, Finite Element Method for Navier-Stokes Equation, Theory and Algorithms. Springer-Verlag, Berlin and Heidelberg (1986).

[9] R.B. Kellogg and J.E. Osborn, A regularity result for the Stokes problem in a polygon. J. Func. Anal. 21 (1976) $397-413$.

[10] Q. Lin, T. Lü and S. Shen, Asymptotic expansions for finite element approximations. Research Report IMS-11, Chengdu Branch of Academia Sinica (1983).

[11] Q. Lin, N. Yan and A. Zhou, A rectangle test for interpolated finite elements in Proc. of Sys. Sci. Es Sys. Eng. Great Wall (H.K.), Culture Publish Co. (1991) 217-229.

[12] Q. Lin and Q. Zhu, The Preprocessing and Postprocessing for the Finite Element Method. Shanghai Scientific \& Technical Publishers (1994) (in Chinese).

[13] G. Marchuk and V. Shaidurov, Difference Methods and their Extrapolation. Springer, New York (1983).

[14] R. Rannacher, Extrapolation techniques in the finite element method (A Survey) in Proc. of the Summer School in Numer. Anal. Helsinki (1988).

[15] R. Teman,Navier-Stokes Equations. North-Holland, Amsterdam (1979).

[16] R. Verfürth, Error estimates for a mixed finite element approximation of the Stokes equations. RAIRO Modèl. Math. Anal. Num. 18 (1984) 175-182.

[17] A. Zhou, Global superconvergence approximations of the mixed finite element method for the Stokes problem and the linear elasticity equation. RAIRO Modèl. Math. Anal. Num. 30, 4 (1996) 401-411.

[18] A. Zhou and J. Li, The full approximation accuracy for the stream function-vorticity-pressure method. Numer. Math. 68 (1994) 427-435.

[19] A. Zhou, C.B. Liem and T.M. Shih, A parallel algorithm based on multi-parameter asymptotic error expansion in Proc. of Conference on Scientific Computing, Hong Kong (1994).

[20] A. Zhou, C.B. Liem, T.M. Shih and T. Lü, A parallel multi-parameter asymptotic error expansion and a parallel algorithm. Research Report IMS-61, Inst. Math. Sci., Academia Sinica (1994), see also Sys. Sci. E Math. Scis. 10, 3 (1997) 253-260. 